\title{
In Vivo Calcium Imaging of CA3 Pyramidal Neuron Populations in Adult Mouse Hippocampus
}

\author{
Gwendolin Schoenfeld, ${ }^{1,2, *}$ Stefano Carta, ${ }^{1,2, *}$ Peter Rupprecht, ${ }^{1}$ Aslı Ayaz, ${ }^{1,2}$ and ${ }^{-}$Fritjof Helmchen ${ }^{1,2}$
}

https://doi.org/10.1523/ENEURO.0023-21.2021

${ }^{1}$ Laboratory of Neural Circuit Dynamics, Brain Research Institute, University of Zurich, Zurich CH-8057, Switzerland and ${ }^{2}$ Neuroscience Center Zurich, University of Zurich, Zurich $\mathrm{CH}-8057$, Switzerland

\begin{abstract}
Neuronal population activity in the hippocampal CA3 subfield is implicated in cognitive brain functions such as memory processing and spatial navigation. However, because of its deep location in the brain, the CA3 area has been difficult to target with modern calcium imaging approaches. Here, we achieved chronic two-photon calcium imaging of CA3 pyramidal neurons with the red fluorescent calcium indicator R-CaMP1.07 in anesthetized and awake mice. We characterize CA3 neuronal activity at both the single-cell and population level and assess its stability across multiple imaging days. During both anesthesia and wakefulness, nearly all CA3 pyramidal neurons displayed calcium transients. Most of the calcium transients were consistent with a high incidence of bursts of action potentials (APs), based on calibration measurements using simultaneous juxtacellular recordings and calcium imaging. In awake mice, we found state-dependent differences with striking large and prolonged calcium transients during locomotion. We estimate that trains of $>30$ APs over $3 \mathrm{~s}$ underlie these salient events. Their abundance in particular subsets of neurons was relatively stable across days. At the population level, we found that co-activity within the CA3 network was above chance level and that co-active neuron pairs maintained their correlated activity over days. Our results corroborate the notion of state-dependent spatiotemporal activity patterns in the recurrent network of CA3 and demonstrate that at least some features of population activity, namely co-activity of cell pairs and likelihood to engage in prolonged high activity, are maintained over days.
\end{abstract}

Key words: auto-associative network; calcium imaging; complex spike burst; hippocampus CA3; juxtacellular; locomotion

\section{Significance Statement}

In vivo measurements of neuronal population activity may reveal how the mammalian hippocampus supports fundamental brain functions such as memory. So far, however, calcium imaging in deep hippocampal regions such as the CA3 subfield has been rarely achieved. Here, we use a red calcium indicator to measure CA3 pyramidal neuron activity in the mouse brain during different states [anesthetized vs awake (resting or running)] and across days. Most CA3 pyramidal neurons displayed calcium transients consistent with complex spike bursts. During running, salient large and prolonged calcium signals were prominent. Some features of neuronal activity remained relatively stable over days, e.g., co-activity in neuronal pairs. Our study further expands CA3 calcium imaging in behaving mice, fostering analysis of CA3 network activity.

\section{Introduction}

Neuronal populations in the hippocampal CA3 subfield are part of the mammalian brain circuit that is essential for spatial navigation, memory formation, and cognition

Received January 15, 2021; accepted June 15, 2021; First published July 30, 2021.

The authors declare no competing financial interests.
(Kesner, 2007; Hartley et al., 2013; Rolls, 2016; Rebola et al., 2017; Hainmueller and Bartos, 2018). CA3 pyramidal neurons are special in forming an auto-associative recurrent network enabling memory encoding and pattern

Author contributions: S.C. and F.H. designed research; G.S. and S.C. performed research; G.S., P.R., and A.A. analyzed data; G.S., P.R., and F.H. wrote the paper. 
completion (Rolls, 2007; Kesner and Rolls, 2015; Guzman et al., 2016; Knierim and Neunuebel, 2016). The functional properties of CA3 pyramidal neurons have been characterized largely with electrophysiology, using extracellular recordings (Fox and Ranck, 1975; Csicsvari et al., 2000; Henze et al., 2002; Leutgeb et al., 2004; Frerking et al., 2005; Mizuseki et al., 2012; Oliva et al., 2016), in vivo intracellular and juxtacellular recordings (Epsztein et al., 2011; Kowalski et al., 2016; Zucca et al., 2017; Diamantaki et al., 2018; Hunt et al., 2018; Malezieux et al., 2020), and whole-cell recordings in brain slices (Jonas et al., 1993; Hemond et al., 2008; Hunt et al., 2018; Raus Balind et al., 2019). Pyramidal neurons in CA3 show properties distinct from CA1 (Mizuseki et al., 2012; Oliva et al., 2016) but display heterogeneity within their population (Hunt et al., 2018; Cembrowski and Spruston, 2019; Ding et al., 2020). For CA3 pyramidal neurons, mean firing rates typically range from $0.3-5 \mathrm{~Hz}$ in vivo (Henze et al., 2002; Wittner and Miles, 2007; Mizuseki et al., 2012; Kowalski et al., 2016; Oliva et al., 2016; Ding et al., 2020), lower than for CA1 pyramidal neurons but higher when compared with dentate gyrus (DG) granule cells. As a prominent feature, hippocampal pyramidal neurons, especially in CA3, exhibit bursts of action potentials (APs) with interspike intervals (ISIs) $<6 \mathrm{~ms}$ (Fox and Ranck, 1975; Frerking et al., 2005; Mizuseki et al., 2012; Kowalski et al., 2016; Oliva et al., 2016; Raus Balind et al., 2019). These complex spike bursts involve regenerative dendritic mechanisms and have been implicated in activity-dependent plasticity (Lee et al., 2012; Grienberger et al., 2014; Bittner et al., 2015, 2017; Diamantaki et al., 2018; Raus Balind et al., 2019). They are also associated with network synchronization events in CA3 (Miles and Wong, 1983; Menendez De La Prida et al., 2006; Wittner and Miles, 2007; Marissal et al., 2012), especially sharp-wave ripples (Buzsáki, 1986; Csicsvari et al., 2000; Harris et al., 2003; Hunt et al., 2018).

Despite these advances in electrophysiological studies, our understanding of CA3 network dynamics and its computational roles remains limited. Optophysiology offers promising complementary approaches, especially in terms of longitudinal imaging of the same neuronal population. However, because of the difficulties in accessing deeper

This work was supported by the Swiss National Science Foundation (SNSF) Projects 31003A_170269, 310030_192617, and Sinergia Project CRSII5-18O316 (to F.H.); the European Research Council (ERC) Advanced Grant BRAINCOMPATH, Project 670757 (to F.H.); a Forschungskredit Postdoc from the University of Zurich (P.R.); and the SNSF Ambizione Grant PZ00P3_161544 (to A.A.).

${ }^{*}$ G.S. and S.C. contributed equally to this work.

A. Ayaz's present address: Neuro-Electronics Research Flanders, 3001 Leuven, Belgium.

Acknowledgements: We thank Lazar Sumanovski for technical assistance and Philipp Bethge, Christopher Lewis, and Xiaomin Zhang for comments on this manuscript.

Correspondence should be addressed to Fritjof Helmchen at helmchen@ hifo.uzh.ch.

https://doi.org/10.1523/ENEURO.0023-21.2021

Copyright (C) 2021 Schoenfeld et al.

This is an open-access article distributed under the terms of the Creative Commons Attribution 4.0 International license, which permits unrestricted use, distribution and reproduction in any medium provided that the original work is properly attributed. brain regions, hippocampal imaging studies have lagged behind similar studies in neocortex. Only during the last decade, in vivo calcium imaging in hippocampus became possible, typically by removing the overlying cortical tissue and using either two-photon microscopy in head-fixed animals (Dombeck et al., 2010; Grienberger et al., 2014; Hainmueller and Bartos, 2018; Kinsky et al., 2018) or miniendoscopes in freely-moving mice (Ziv et al., 2013; Rubin et al., 2015; Gonzalez et al., 2019; Stefanini et al., 2020). While initial studies mainly targeted CA1 as the most accessible region, only at a later stage chronic and functional imaging was also established in the DG (Pilz et al., 2016, 2018; Danielson et al., 2017; Hainmueller and Bartos, 2018; Stefanini et al., 2020). In our own previous study (Pilz et al., 2016), by applying GCaMP6 and specifically RCaMP1.07, a red calcium indicator that facilitates deep imaging (Ohkura et al., 2012; Bethge et al., 2017), we confirmed sparse activity of DG granule cells and described its variation across behavioral states. Functional imaging in CA3 is as challenging as in DG and therefore has been achieved in only few studies until today (Rajasethupathy et al., 2015; Hainmueller and Bartos, 2018; Rashid et al., 2020). As an emerging field, CA3 imaging provides new opportunities to address key questions about cellular and circuit mechanisms of neural coding and plasticity in this region.

Here, we establish in vivo calcium imaging of CA3 pyramidal neurons using an approach similar to our previous DG study (Pilz et al., 2016). We characterize basic features of CA3 calcium transients and calibrate them in terms of underlying APs using simultaneous juxtacellular recordings. We find heterogeneous CA3 activity patterns across behavioral states and discover particularly prominent prolonged calcium transients that occur in neuronal subsets during running. Moreover, our longitudinal imaging results indicate that CA3 population activity at least partially remains stable across days, particularly with respect to the co-activity of neurons within sub-ensembles.

\section{Materials and Methods}

\section{Animals and R-CaMP1.07 labeling}

All experimental procedures were conducted in accordance with the ethical principles and guidelines for animal experiments of the Veterinary Office of Switzerland and were approved by the Cantonal Veterinary Office in Zurich. For the experiments, male and female mice with a $\mathrm{Tg}$ (Grik4-cre)G32-4Stl background were used $(n=6)$. These mice show a dense expression of Cre-recombinase rather specific to CA3 hippocampal pyramidal neurons (MGI:2387441; Nakazawa et al., 2002). We induced expression of the red fluorescent calcium indicator RCaMP1.07 (Ohkura et al., 2012) in CA3 pyramidal neurons by stereotaxic injection of AAV1-EF $\alpha 1$-DIO-R-CaMP1.07 in six- to nine-week-old adult mice (coordinates: AP -2 , $\mathrm{ML}+1.8$, DV -2.2 ; in $\mathrm{mm}$ from bregma; $300 \mathrm{nl}$ with a virus titer of $\left.\sim 1 \times 10^{7} \mathrm{vg} / \mathrm{hl}\right)$.

\section{Hippocampal window implantation}

Chronic access for CA3 imaging was obtained by the implantation of a hippocampal window (Pilz et al., 2016). 
One week after the virus injection, we performed a 3-mm diameter craniotomy centered at the injection site and implanted a stainless-steel cannula with a front glass window. After removing the bone, we gently aspirated the underlying cortical tissue until the corpus callosum fibers became visible. A stainless-steel cannula $(\varnothing 3 \mathrm{~mm}, 1.5$ $\mathrm{mm}$ length) covered by a glass coverslip ( $\varnothing 3 \mathrm{~mm}, 0.17-$ $\mathrm{mm}$ thickness) was inserted into the cavity and secured in place using dental acrylic cement (Ivoclar Vivadent; Fig. $1 A, B)$. Additionally, an aluminum post for head fixation during imaging was attached to the skull. After a recovery period, mice were handled by the experimenter, habituated to head fixation, and accustomed to run on a ladder wheel $(\varnothing 23 \mathrm{~cm})$ with regularly spaced rungs $(1-\mathrm{cm}$ spacing) during head fixation. Approximately two weeks after the surgery, neuronal population activity was imaged under isoflurane anesthesia (1-2\% in oxygen) on three to five consecutive days. The same neuronal populations that were imaged in the anesthetized condition were repeatedly imaged in awake animals for $5-10 \mathrm{~d}$, of which at least $3 \mathrm{~d}$ were consecutive (Fig. 1C).

\section{Two-photon calcium imaging}

We used a custom-built two-photon microscope based on the Sutter movable objective microscope (MOM) type, equipped with a water immersion $16 \times$ objective (CFI LWD $16 \times / 0.80$; Nikon), a Pockels cell (model 350/80 with controller model 302RM, Conoptics), and galvanometric scan mirrors (model 6210; Cambridge Technology), controlled by HelioScan software (Langer et al., 2013). R-CaMP1.07 was excited by $\sim 230$-fs pulses at $80 \mathrm{MHz}$ provided by a ytterbium-doped potassium gadolinium tungstate ( $\mathrm{Yb}$ : KGW) laser (1040 nm; >2-W average power; model Ybix; Time-Bandwidth Products). Emitted fluorescence was detected by a photomultiplier tube after passing through a 610/75-nm bandpass filter (AHF Analysetechnik). Laser intensities during imaging were $56-78 \mathrm{~mW}$ under the objective.

In anesthetized experiments, mice were anesthetized with isoflurane $\left(1-2 \%\right.$ in $\left.\mathrm{O}_{2}\right)$. Body temperature was monitored continuously with a thermosensor and kept at $37^{\circ} \mathrm{C}$ with a heating blanket. For awake experiments, the headfixed mouse was placed on the ladder wheel and was free to run. Running speed and running distance during calcium imaging were recorded at $40 \mathrm{~Hz}$ with a rotary encoder (Phidgets, 12V/0.2Kg-cm/230RPM 10:1 DC gear motor with encoder). The activity of R-CaMP1.07-expressing CA3 pyramidal cells was recorded in trials of 30-s duration, with 10-s inter-trial intervals (maximum of 30 trials per day). Recordings were performed in the distal part of CA3 (CA3a), which lays in the proximity of CA2. In all sessions, imaging across a field of view (FOV) of $325 \times 325 \mu \mathrm{m}^{2}$ was performed at $10-\mathrm{Hz}$ frame rate.

\section{In vivo electrophysiology}

Electrophysiological recordings, combined with in vivo calcium imaging, were performed in acute in vivo preparations of Tg(Grik4-cre)G32-4Stl expressing R-CaMP1.07 mice ( $n=3$; at least two weeks after injection). Mice were anesthetized with isoflurane and the temperature was maintained at $37^{\circ} \mathrm{C}$. A stainless steel plate was fixed to the exposed skull using dental acrylic cement. A 4-mm diameter craniotomy was performed, centered above the virus injection locus. The overlying cortex was aspirated until the corpus callosum became visible. A 1\%-agarose gel was filled into the cavity to reduce tissue motion. Juxtacellular recordings from R-CaMP1.07-expressing CA3 pyramidal neurons were obtained with glass pipettes (4- to 6-M $\Omega$ pipette resistance) filled with Ringer's solution. To facilitate visually-guided targeting of individual neurons, the pipette was coated with BSA Alexa Fluor 594 (Invitrogen). APs were recorded juxtacellularly in current clamp mode at $10-\mathrm{kHz}$ sampling rate using an Axoclamp 2B amplifier (Molecular Devices, Molecular Devices) and digitized using Clampex 10.2 software. Simultaneously, we performed two-photon calcium imaging at $20-\mathrm{Hz}$ frame rate, zoomed-in on the recorded neuron to optimize signal-to-noise ratio.

\section{Perfusion and histology}

After the last awake imaging session, mice were administered a lethal dose of pentobarbital (Ekonarcon, Streuli) and transcardially perfused with sterile $\mathrm{NaCl}(0.9 \%)$ followed by $4 \%$ paraformaldehyde (PFA; $0.1 \mathrm{M}$ phosphate buffer, $\mathrm{pH}$ 7.4). We cut 40- $\mu \mathrm{m}$ coronal brain slices and acquired histologic images with a confocal laser-scanning microscope (Olympus FV1000) using 546-nm laser light for R-CaMP1.07 excitation (Fig. 1B).

\section{Data analysis}

Electrophysiological data were analyzed using routines in IGOR (Wavemetrics). R-CaMP1.07 fluorescence signals were analyzed using custom-written macros in ImageJ (Schindelin et al., 2012) and MATLAB routines (The MathWorks). For motion correction of calcium imaging movies, we applied a hidden Markov model line-by-line motion correction algorithm (Dombeck et al., 2007). We excluded trials that obviously were insufficiently motioncorrected based on visual inspection. Regions of interest (ROIs) corresponding to individual neurons were manually selected from the mean fluorescence image of a singletrial time series. Background fluorescence was estimated as the bottom first percentile fluorescence signal across the entire session and subtracted before calculating the relative percentage fluorescence change from baseline $\Delta \mathrm{F} / \mathrm{F}=\left(\mathrm{F}-\mathrm{F}_{0}\right) / \mathrm{F}_{0}$. Baseline fluorescence $\mathrm{F}_{0}$ was computed as 51st percentile of the fluorescence signal in an 8-s sliding window. $\Delta \mathrm{F} / \mathrm{F}$ traces were smoothed with a five-point first-order Savitsky-Golay filter.

For characterization of R-CaMP1.07 signals based on ground truth data (Fig. 2), we aligned the simultaneously recorded electrophysiological traces and fluorescence signals at the start of recording. We determined the peak amplitude of isolated calcium transients (i.e., with no spiking activity in a 2-s period before the first AP associated with the calcium transient) and counted the number of underlying APs. To focus on quasi-impulse-like responses, we only considered transients with APs that 
A

C

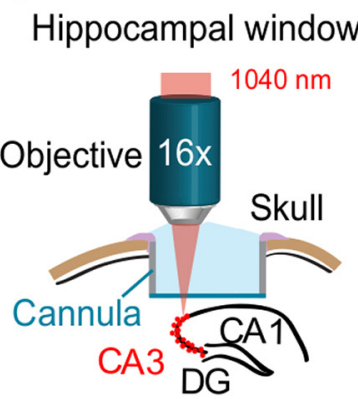

Girk4-Cre mouse $(n=6)$

D Anesthetized

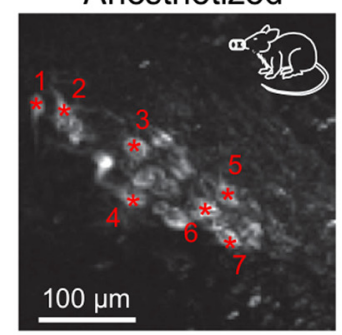

E

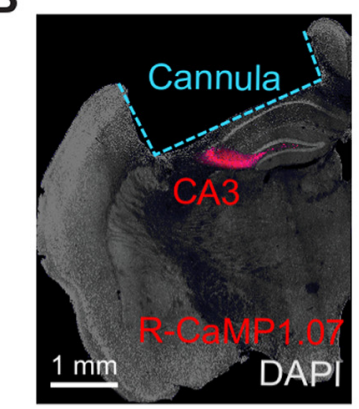

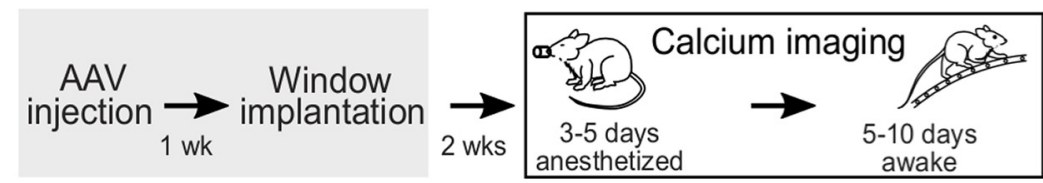
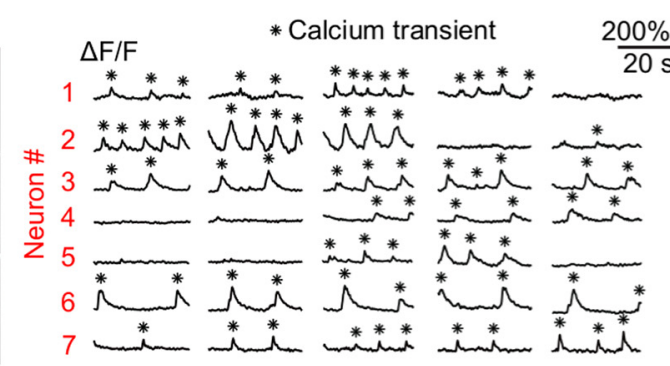

* Calcium transient
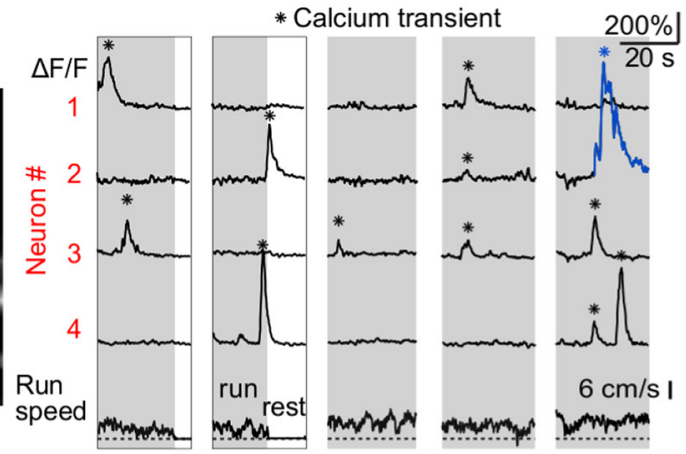

Figure 1. In vivo two-photon imaging of CA3 pyramidal neuron populations during wakefulness and anesthesia. $\boldsymbol{A}$, Schematic illustration of experimental setup showing the chronic window implant above the corpus callosum and area CA1 of the intact hippocampus for R-CaMP1.07 calcium imaging in CA3. B. Histologic coronal cross-section of the fixed brain after in vivo imaging sessions showing R-CaMP1.07-labeled CA3 pyramidal neurons and the hippocampal window implant. C, Experimental time line. After recovery from the surgery, three consecutive days of anesthetized imaging were conducted. This was followed by $6-10 \mathrm{~d}$ of awake imaging including at least three consecutive imaging days. $\boldsymbol{D}$, Example FOV of an anesthetized two-photon imaging session. Example neurons are labeled by red asterisks and their respective $\Delta \mathrm{F} / \mathrm{F}$ traces are displayed on the right. Detected calcium transient peaks are labeled with black asterisks. $\boldsymbol{E}$, Example FOV and $\Delta \mathrm{F} / \mathrm{F}$ traces of neurons recorded during wakefulness. An example large calcium transient is labeled in blue. Run speed of the animal is shown below.

occurred within a time window of maximally 200 -ms duration. For averaging, calcium transients were aligned to the first AP of a given event.

We also used the ground truth dataset $(n=4$ neurons from three mice; a total of $33 \mathrm{~min}$ of recording and 5025 APs) to train a supervised algorithm based on neural networks to deconvolve calcium transients and estimate the underlying spike rates (SRs). The deconvolution algorithm, which we present in detail in a separate paper (Rupprecht et al., 2021), was trained on the R-CaMP1.07 ground truth data, which were re-sampled to the $10-\mathrm{Hz}$ frame rate used for awake imaging. The noise level of the ground truth data was adjusted to match the noise level of each neuron of the population imaging data by adding Poisson noise. Spike trains used to train the network were temporally filtered with a Gaussian [ 470-ms full-widthhalf-maximum (FWHM)]. Prediction of SRs using this approach is expected to show correlation values of $0.79 \pm$ 0.16 (mean \pm SD) with the ground truth data, thus explaining $\sim 60 \%$ of the variance (Rupprecht et al., 2021).

For analysis of population imaging data in Figures 3, 4, we defined detectable calcium transients as fluorescence signals that deviated from baseline by $>3 \mathrm{SD}$ for anesthetized imaging and $>4$ SD for awake conditions. We applied the more stringent criterion for awake conditions because of increased noise levels and possible motion artifacts during wakefulness. For every thresholdcrossing event we determined the calcium transient peak as the first maximum found by the MATLAB function findpeaks (using minimal peak prominence of $20 \% \Delta \mathrm{F} / \mathrm{F}$ and minimal peak separation of $1.5 \mathrm{~s}$ ). We then excised 3-s segments around the detected calcium transient events $(-1$ to $+2 \mathrm{~s}$ relative to the peak) and estimated the underlying SRs using the deconvolution algorithm. For each event, we computed the mean $\Delta \mathrm{F} / \mathrm{F}$ value in the 3-s time window, reflecting the integral cellular activity causing the calcium transient. For the awake recordings, we defined "large" calcium transients as those that displayed mean $\Delta \mathrm{F} / \mathrm{F}$ values larger than the 95th percentile of the distribution of mean $\Delta \mathrm{F} / \mathrm{F}$ values for all transients recorded during anesthesia. The ground truth data recorded during anesthesia did not fully cover calcium transient amplitudes and shapes representative of the large and prolonged calcium transients observed during wakefulness. To estimate the number of APs during these calcium transients in Figure 3, we therefore used a model-free 

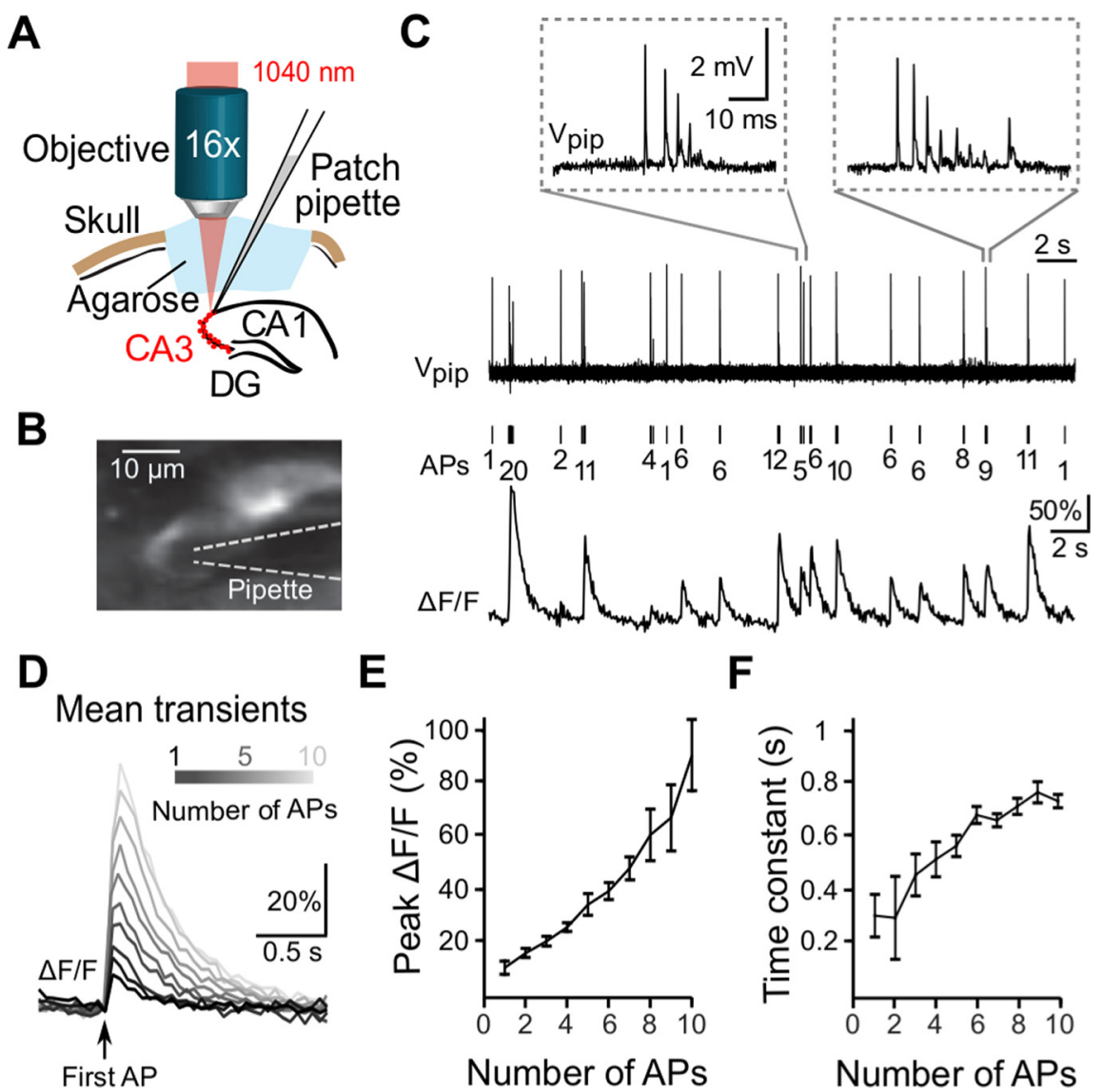

Figure 2. Calibration of in vivo spiking activity of CA3 pyramidal neurons using simultaneous two-photon calcium imaging and juxtacellular voltage recordings in acute experiments. $\boldsymbol{A}$, Schematic of experimental setup. $\boldsymbol{B}$, Maximum-intensity projection of two-photon images of R-CaMP1.07-labeled CA3 pyramidal neurons together with the recording pipette in juxtacellular configuration. $\boldsymbol{C}$, Example traces of simultaneously recorded calcium transients $(\Delta \mathrm{F} / \mathrm{F})$ and spontaneous $A P s\left(V_{\text {pip }}\right)$ during isoflurane-anesthesia. The number of APs per burst is indicated below. Insets show two example burst events magnified. $\boldsymbol{D}$, Average calcium transients caused by 1-10 APs (within $200 \mathrm{~ms}$ ) aligned to the occurrence of the first AP. $\boldsymbol{E}$, Relationship between peak $\Delta \mathrm{F} / \mathrm{F}$ changes and number of APs (mean \pm SEM). $\boldsymbol{F}$, Relationship between the calcium transient decay time constant and the number of APs (exponential fits; error bars indicate $90 \%$ confidence interval). The heterogeneity of burst events is further analyzed in Extended Data Figure $2-1$.

look-up table based on the integral of the SR predictions in the excised 3-s calcium transient segment (Fig. 3B, bottom). A 95\% confidence corridor for the data was obtained by Gaussian process regression (with the MATLAB function fitrgp), using squared exponentials as the kernel functions and optimizing hyperparameters of the Gaussian process regression with cross-validation. For extra analysis based on the deconvolved calcium transients, we detected peaks of the estimated SR trace with a similar procedure as for $\Delta F / F$ traces (minimal peak prominence $1.5 \mathrm{~Hz}$, minimal peak separation $1.5 \mathrm{~s}$ ).

For analysis of neuronal population activity under anesthetized, awake resting, and awake running conditions, we identified in each FOV the neurons that were co-active with the events detected in a reference neuron. Co-activity was defined as showing a calcium transient peak within a 1-s window surrounding the peak of the reference event ( $0.5 \mathrm{~s}$ before to $0.5 \mathrm{~s}$ after). The percentage of coactive neurons per event of all neurons analyzed in the FOV was determined separately for anesthetized and awake conditions. Results were compared with shuffled co-activity values obtained by randomizing the peak times of detected calcium transients in tested neurons (taking the mean of 100 randomizations). To evaluate the statistical difference between conditions we subtracted the mean values obtained from shuffled data and then computed the ratio between two conditions, e.g., awake resting versus running. We estimated the SEM by Gaussian error propagation.

Additionally, we assessed the stability of co-activity of neuron pairs over multiple days. For each day, for which a pair of neurons (e.g., neurons A and B) was recorded, we determined the fraction of co-active events compared with all events in these neurons. Co-activity again was -defined by the co-occurrence of an event in neuron $B$ within a 1-s window around a detected event in reference neuron $A$ and vice versa for reference events in neuron $B$ (resulting in a symmetric co-activity measure by taking the mean). For the shuffle control, we randomized peak times of all events in the non-reference neurons (100 times repeated per neuron pair). Stability was assessed by the Pearson's correlation coefficient $(\rho)$ comparing co- 
activity values on $1 \mathrm{~d}$ (day $N+1$ ) with the previous day $(N)$. We also repeated the co-activity analysis for the deconvolved calcium transients (SR traces), which did not change the results. To probe the robustness of the results against the choice of the time window, we tested additional time windows from 0.1 - to 4.5 -s duration (Extended Data Fig. 4-2A). To analyze the dependence of co-activity values on the distance between neuron pairs, we used the Euclidean distances between the centroids of the respective ROls (Extended Data Fig. 4-3).

\section{Run speed analysis}

We down-sampled running speed to the $10-\mathrm{Hz}$ imaging frame rate and defined periods with $>0.5 \mathrm{~cm} / \mathrm{s}$ speed as "run" periods and periods with lower speeds as "rest" periods. The numbers of small and large calcium transients per minute during wakeful resting or locomotion were determined and distributions were compared using one-way ANOVA. The normalized frequency distributions of wakeful small and large events across run speeds were compared using a Kolmogorov-Smirnov test.

\section{Results}

\section{In vivo two-photon calcium imaging of CA3 pyramidal neurons}

We established in vivo two-photon imaging of CA3 neuronal population activity through a chronically implanted window after removal of cortical tissue overlying the hippocampus (Fig. 1A,B). To induce expression of a genetically encoded calcium indicator specifically in CA3 pyramidal neurons, we injected Grik4-Cre transgenic mice with a virus driving Cre-dependent expression of the red-shifted calcium indicator R-CaMP1.07 (Ohkura et al., 2012; Bethge et al., 2017; Fig. 1B). Following chronic window preparation and habituation of the mouse to headfixation, we performed calcium imaging of R-CaMP1.07expressing CA3 pyramidal neurons in several sessions of around $30 \mathrm{~min}$ in duration, spread over consecutive days and under either anesthetized or awake condition (Fig. $1 C)$. During awake recordings, mice were free to run or rest on a ladder wheel placed under the two-photon microscope. We continually measured running speed and used a threshold to distinguish behavioral states by defining run and rest periods.

Nearly all neurons exhibited calcium transients indicative of neuronal spiking activity in both anesthetized and awake condition (Fig. $1 D, E)$. For every mouse $(n=6)>90 \%$ of cells showed at least one detectable calcium transient on the first imaging day with isoflurane-anesthesia (90\%, 124 cells, six FOVs) as well as on the first awake imaging day (93\%, 234 cells, 10 FOVs; for criteria for detection of calcium transients, see Materials and Methods). Calcium transients occurred rather regularly in individual neurons in anesthetized mice. In contrast, amplitudes and durations of calcium transients were more heterogeneous in awake mice, including a substantial fraction of large and prolonged events (an example is colored in Fig. 1E). On average, calcium transients were smaller and of shorter duration during anesthesia compared with wakefulness $(\Delta F / F$ peak amplitude $45.0 \pm 26.3 \%$ vs $89.5 \pm 65.0 \%$; FWHM $1.8 \pm 2.3$ vs $2.3 \pm 2.1 \mathrm{~s}$; mean \pm SD, 2934 transients in 138 neurons for anesthetized and 2806 transients in 251 neurons for awake condition; $p<0.001$ for both comparisons; twosided Wilcoxon rank-sum test). These results indicate that CA3 pyramidal neurons show distinct patterns of neuronal activity in anesthetized compared with awake condition.

\section{Juxtacellular recordings of R-CaMP1.07-expressing CA3 pyramidal neurons}

To relate R-CaMP1.07 calcium transients to AP patterns we performed acute experiments in anesthetized mice, obtaining simultaneous juxtacellular recordings and functional calcium imaging data from R-CaMP1.07expressing CA3 pyramidal neurons (Fig. 2A,B). We extracted spike times using simple thresholding and temporally aligned calcium transients to the voltage recordings. Juxtacellular recordings revealed APs in variable numbers, often occurring in high-frequency bursts (Fig. 2C). The amplitude of consecutive spikes within a burst decreased over four to six APs, until no more spikes could be detected. For longer bursts, the AP amplitude often partially recovered after this initial decrease (Fig. 2C; Extended Data Fig. 2-1A,B). Burstiness was apparent in the bimodal distribution of ISIs, with two peaks at $5.3 \pm 7.3 \mathrm{~ms}$ and $0.81 \pm 1.51 \mathrm{~s}$ (mean $\pm \mathrm{SD}$ ), reflecting intraburst and interburst intervals, respectively (Extended Data Fig. 2-1C).

AP patterns in individual neurons correlated with the measured calcium transients (Fig. 2C). A spontaneous single $A P$-evoked $\Delta F / F$ transient on average had a peak amplitude of $11 \pm 3 \%$ ( $n=47$ events, four neurons, three mice). With increasing number of APs, the $\Delta F / F$ amplitude of the corresponding calcium transients increased, following an approximately linear relationship up to 10 APs (Fig. 2D,E). The decay time constant of the evoked transient, as measured by an exponential fit, was around $0.3 \mathrm{~s}$ for single APs and remained $<0.8 \mathrm{~s}$ for larger numbers of APs (Fig. 2F). These ground-truth data provide an important calibration resource that helps to interpret $\mathrm{R}$ CaMP1.07 imaging data in CA3 neuronal populations more quantitatively.

\section{Large and prolonged calcium transients during wakefulness and locomotion}

Taking advantage of this ground-truth calibration, we trained a supervised spike inference algorithm based on a deep neuronal network to temporally deconvolve $\Delta \mathrm{F} / \mathrm{F}$ transients and infer instantaneous SRs (Rupprecht et al., 2021; Materials and Methods). Deconvolution uncovered that during wakefulness, in contrast to anesthesia, calcium transients often were prolonged, indicating extended periods of spiking, sometimes over seconds (Fig. $3 A$ ). For quantification, we computed the mean $\Delta F / F$ value in a 3-s time window around the peak of a detected calcium transient ( $1 \mathrm{~s}$ before until 2 $s$ after the peak), reflecting the integral cellular activity (overall number of APs) causing the calcium transient. The distribution of mean $\Delta \mathrm{F} / \mathrm{F}$ values was significantly 
A Deconvolution of $\Delta \mathrm{F} / \mathrm{F}$ traces

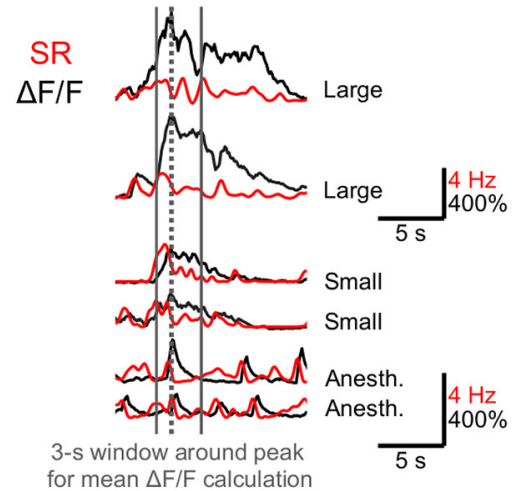

C

Mean calcium transients

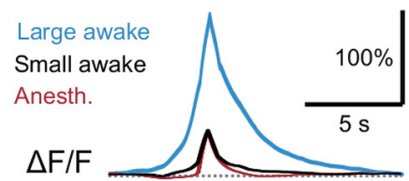

D

Small transients Large transients

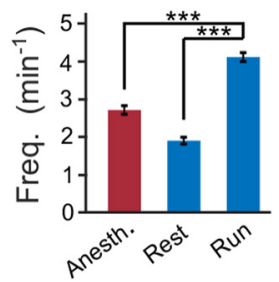

B

$\Delta F / F$ amplitude distribution
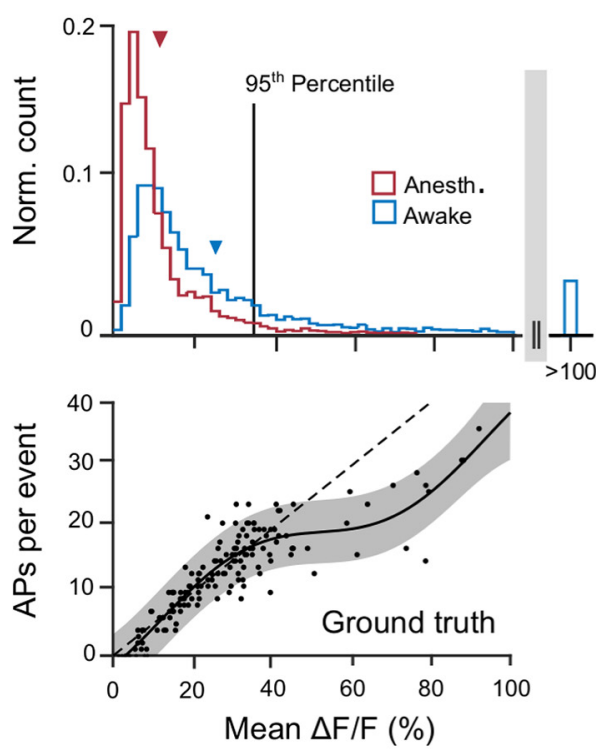

E

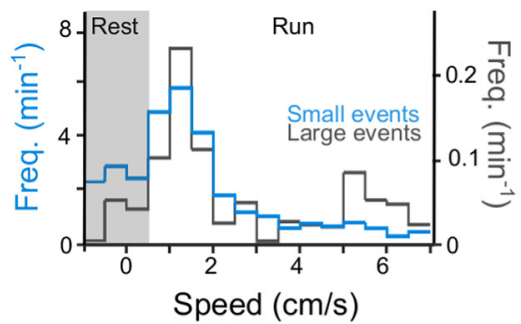

Figure 3. Large and prolonged calcium events in CA3 pyramidal neurons during wakefulness. $\boldsymbol{A}$, Example $\Delta \mathrm{F} / \mathrm{F}$ traces (black) with corresponding estimated spike rates (SR, red). $\boldsymbol{B}$, Distribution of mean $\Delta \mathrm{F} / \mathrm{F}$ level in a 3-s time window around calcium transient peaks (dashed line in $\boldsymbol{A}$ ) under anesthesia (red) and during wakefulness (blue). Wakeful transients that exceeded the 95th percentile of anesthetized mean $\Delta F / F$ levels $(37 \%$ mean $\Delta F / F)$ were classified as large events. Arrows indicate the respective mean of each distribution (awake: $23 \pm 24 \%$, anesthetized: $11 \pm 10 \%$, mean \pm SD, $p<0.001$, two-sided Wilcoxon rank-sum test). The lower panel, as a calibration look-up table for the upper panel, shows the relation of mean $\Delta F / F$ level to the number of APs extracted from the ground-truth dataset. The linear reference line is fitted to data points with mean $\Delta \mathrm{F} / \mathrm{F}$ values $<40 \%$. The $95 \%$ confidence corridor (gray shading) is based on a model-free fit of all data points. $\boldsymbol{C}$, Average shape of calcium transients classified as anesthetized events, awake small and awake large events (mean \pm SEM). $\boldsymbol{D}$, left, Mean frequency of small calcium transients per neuron and session under anesthetized, awake resting, and awake running conditions (mean \pm SEM; resting vs anesthetized: $0.70 \pm 0.04$, running vs anesthetized: $1.53 \pm 0.08$, running vs resting: $2.19 \pm 0.12$; ratio of means \pm error propagation of SEM). Right, Mean frequency of large calcium transients across neurons during awake resting and awake running condition (running vs resting: $3.67 \pm 0.83$; ratio of means \pm error propagation of SEM); ${ }^{* *} p<0.001$, one-way ANOVA. E. Frequency distribution of 1859 small awake events (blue) and 947 large awake events (black), normalized to the times spent at specific speeds, as a function of running speed.

shifted to higher values during wakefulness compared with anesthesia $(23 \pm 24 \%$ vs $11 \pm 10 \%$, mean \pm SD, $p<0.001$, two-sided Wilcoxon rank-sum test; Fig. $3 B$ ), in qualitative agreement with recent findings in CA1 (Yang et al., 2021). The distribution of mean $\Delta F / F$ values for the awake condition showed a pronounced tail of large events, with a substantial fraction reaching $>100 \%$ mean $\Delta F / F$. To account for these special events, we defined "awake large" events as those calcium transients with mean $\Delta F / F$ values larger than most anesthetized events ( $>95$ th percentile; Fig. $3 B$ ). According to this definition, $33.7 \%$ of all awake events were classified as large events. Overall, we classified our recorded calcium transients in "anesthetized" ( $n=2934)$, "awake small" $(n=1859)$, and "awake large" $(n=947)$ events. We did not further divide calcium transients that were measured during anesthesia into small and large transients. The average shape and amplitude of anesthetized calcium transients resembled the small awake events, whereas the awake large events exhibited higher amplitudes and prolonged durations (Fig. $3 C$ ).

To further evaluate AP patterns that underlie detectable calcium transients as shown in Figure $3 A-C$, we performed additional analyses on the ground truth. For every transient detected according to our criteria, we analyzed the AP patterns in the 3-s window around the transient peak and compared it to AP firing patterns in time periods 
A

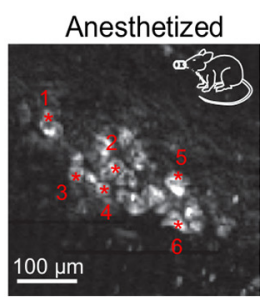

B

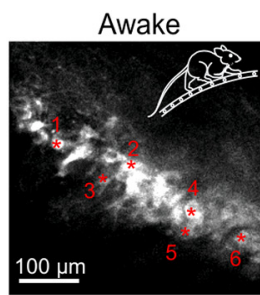

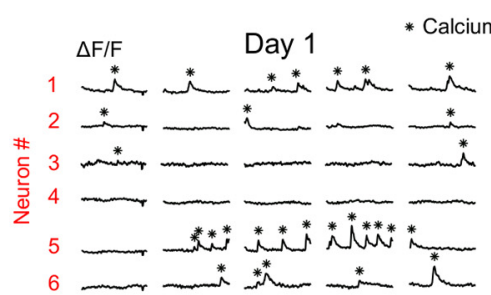

Day 1

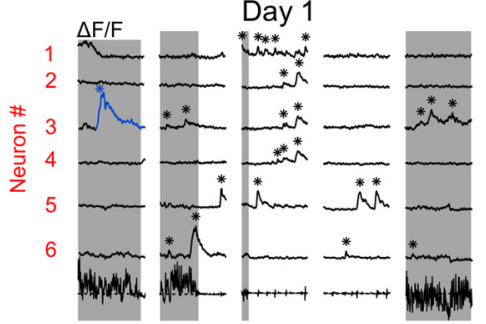

Width

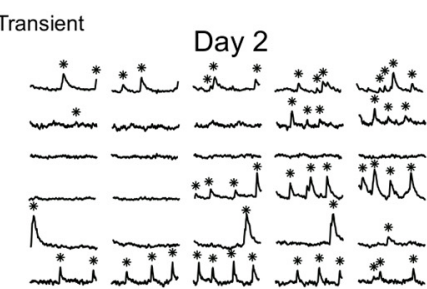

Day 2

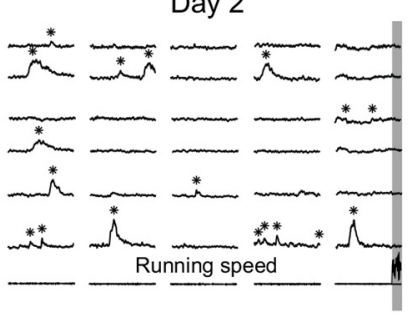

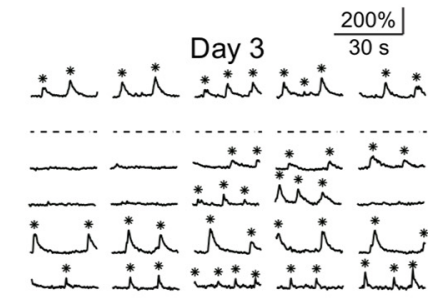

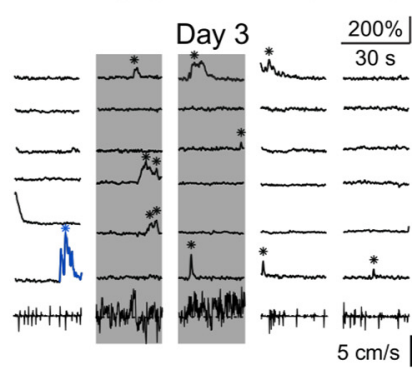

E
C

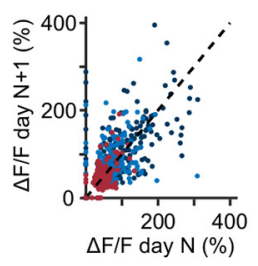

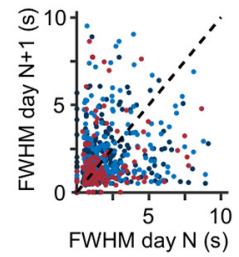

- Awake Rest
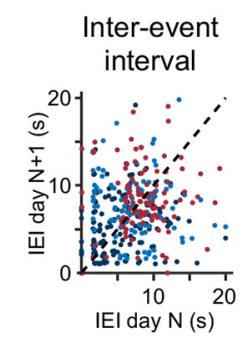

D

Cells with large events $(n=47)$

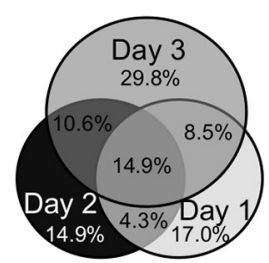

Percent of co-active cells per event

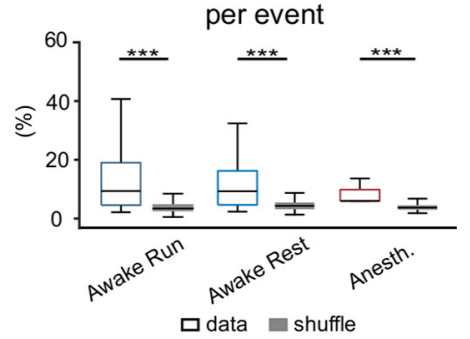

$\mathbf{F}$

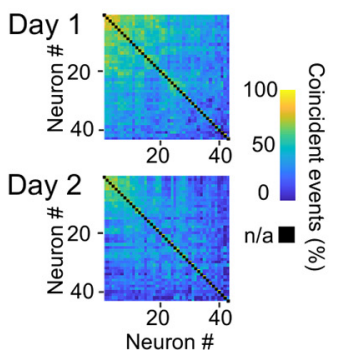

G

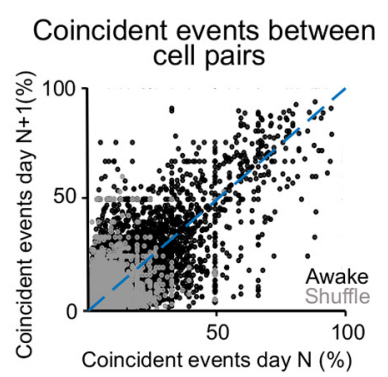

H 2D point density

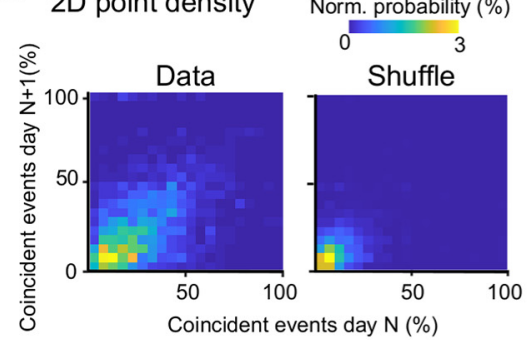

Figure 4. Longitudinal functional imaging of CA3 population activity over consecutive recording days. $\boldsymbol{A}$, Example FOV (left) and calcium transients of selected neurons over three consecutive imaging days during anesthesia. $\boldsymbol{B}$, Example FOV and across-days calcium transient examples during wakefulness. Example large calcium events are labeled blue. Periods of locomotion are highlighted in gray. $\boldsymbol{C}$, Comparison of mean $\Delta \mathrm{F} / \mathrm{F}$ transient amplitude, mean FWHM, and mean IEl per neuron over consecutive imaging days (dashed lines represent unity lines; for Pearson correlations see main text). For a plot of these calcium transient properties across all neurons and days, see Extended Data Figure 4-1. D, Percentage of cells firing large events for each day and over consecutive days (number of neurons: $\left.n_{\text {day } 1}=21, n_{\text {day } 2}=21, n_{\text {day } 3}=30, n_{\text {day } 1+\text { day } 2}=9, n_{\text {day } 2+\text { day } 3}=12, n_{\text {day } 1+\text { day } 3}=11, n_{\text {day } 1+\text { day } 2+\text { day } 3}=7\right)$. $\boldsymbol{E}$, Percentage of neurons in a FOV that show co-activity within a 1-s time window surrounding a detected event, compared with randomly shuffled event times $\left({ }^{* * *} p<0.001\right.$; box displays median and 25th and 75th percentiles, whiskers indicate first and 99th percentiles; resting vs anesthetized $2.15 \pm 0.05$, running vs anesthetized $2.57 \pm 0.06$, resting vs running $1.20 \pm 0.02$; ratio of means \pm error propagation of SEM). $\boldsymbol{F}$, Percentage of coincident events between all neuron pairs of one example FOV over $2 \mathrm{~d}$. Hierarchical link clustering was performed on the co-activity matrix of day 1 , and the resulting order of neurons was maintained for day 2 . $\mathbf{G}$, Comparison of percentage of coincident events per neuron pair over consecutive imaging days. For this plot all data from all FOVs were pooled (experimental data: $\rho=0.34$, shuffled data: $\rho=0.06$ ). Dashed line represents unity line. $\boldsymbol{H}$, Normalized 2D density plot based on $\boldsymbol{G}$. For temporal aspects of co-activity and its dependence on neuron pair distance see Extended Data Figures 4-2, 4-3, respectively.

without detectable calcium transients. Detected calcium transients were induced by bursts of more than three APs $(6.4 \pm 4.4$ APs without interruption; mean \pm SD; bursts defined as APs with ISls of $<10 \mathrm{~ms}$, according to the histogram in Extended Data Fig. 2-1C), indicating that our analysis misses a "floor" of single APs or very brief bursts that are hidden in the noise (we estimate this fraction could be as large as 30\%). To permit a more direct interpretation of calcium signals in terms of underlying spikes, we generated a look-up table for the number of APs versus the mean $\Delta F / F$ value in the 3-s analysis window from the calcium transients detected in our ground-truth 
dataset (Fig. 3B, bottom). This relationship was approximately linear for small $(<40 \%)$ mean $\Delta F / F$ values and tapered off at higher values. Note that this tapering-off corresponds to a supra-linear increase of mean $\Delta \mathrm{F} / \mathrm{F}$ values with the number of APs, possibly reflecting additional calcium influx caused by regenerative dendritic events associated with AP bursts (Grienberger et al., 2014; Raus Balind et al., 2019). Because large calcium transients with $>40 \%$ mean $\Delta F / F$ were rare under anesthesia, this observation is based on only few data points, however, and therefore needs to be interpreted carefully. The variability of the estimated number of APs increased at high mean $\Delta F / F$ values, presumably indicating variations of the temporal profile of the underlying spike trains. Applying this look-up table to calcium transients measured during wakefulness, we estimate that small events reflect short bursts of APs or trains of up to 20 APs whereas the largest events with $>100 \%$ mean $\Delta \mathrm{F} / \mathrm{F}$ presumably were caused by $>30$ APs within the 3 -s window (Fig. 3B, top). As a limitation to this approach, it must be kept in mind that AP patterns, i.e., bursting versus continuous spiking, are not necessarily preserved between anesthetized and awake states.

The abundance of small calcium transients was comparable during anesthesia and awake resting condition but higher for awake running; moreover, large calcium transients occurred almost exclusively during running (Fig. 3D). Although the frequency distributions of small and large events across running speed were not significantly different $(p=0.64$, Kolmogorov-Smirnov test), there was a trend for large events to particularly occur at the highest speeds (Fig. 3E). In summary, we observed especially large-amplitude calcium transients with prolonged duration during wakefulness, in particular during running.

\section{Stability and variability of neuronal activity and co-activity in CA3 across days}

To assess how stable or variable the activity of CA3 pyramidal neurons is over days, we analyzed calcium transients measured repeatedly in the same neuronal populations over three consecutive days in both anesthetized and awake state (Fig. $4 A, B$ ). In the awake condition, 181 out of 251 neurons could be tracked across all $3 \mathrm{~d}$ (72\%); in anesthesia sessions, 91 out of 138 neurons $(66 \%)$ were consistently tracked. For each neuron, we calculated the mean $\Delta \mathrm{F} / \mathrm{F}$ peak amplitude, the average interevent interval (IEI) time and the average width (FWHM) for all calcium transients per day (Extended Data Fig. 4-1A, $C)$. We quantified the stability of these features by correlating values recorded during one imaging day $N+1$ with values for the same neurons from the previous day $N$ (Lütcke et al., 2013; Fig. 4C). While the $\Delta F / F$ amplitude for the same neurons was relatively stable across days (Pearson's correlation coefficient $\rho=0.50,0.34$, and 0.54 for anesthetized, awake resting, and awake running condition, respectively; all $\left.p<6 \times 10^{-7}\right)$, correlation values were lower for FWHM $(\rho=0.20,0.09$, and 0.15 , respectively; with $p=0.01,0.23,0.02)$ and IEI ( $\rho=0.16,0.04$, and 0.17 , respectively; with $p=0.09$,
$0.65,0.04)$. Motivated by the observation that $\Delta \mathrm{F} / \mathrm{F}$ amplitudes were relatively stable, we specifically addressed the question how the distribution of large events (as defined in Fig. 3) changed over days across the population. In a subset of the neurons tracked across $3 \mathrm{~d}$ (47 out of 181 neurons) we observed large events on at least $1 \mathrm{~d}$ (33.7\% of events in total). About a third of these neurons (33.4\%) displayed large events on at least $2 \mathrm{~d}$ (chance level 18.2\%; $p=0.012$; Monte Carlo simulation of the null distribution) and a considerable fraction (14.9\%) even on all three consecutive measurement days (chance level $0.85 \% ; p<1 \times 10^{-6}$; Fig. $4 D$ ). These above-chance incidences indicate that a subset of neurons exists that is particularly prone to generate large events consistently over days.

Finally, we investigated CA3 neuronal activity on the population level. To assess synchrony of activity we calculated the percentage of co-active neurons per calcium transient per FOV, with co-activity defined as co-occurrence of calcium transients in a 1-s time window ranging from $0.5 \mathrm{~s}$ before to $0.5 \mathrm{~s}$ after an event (Materials and Methods; see also Extended Data Fig. 4-2A-C for variable time windows and transient peak time distribution of coactive transients). The percentage of co-active neurons per event was significantly higher than expected from chance level for all conditions $(6.3 \pm 3.6 \%, 14.1 \pm 11.8 \%$, and $12.4 \pm 10.7 \%$ for anesthetized, awake running, and awake resting, respectively; mean \pm SD; $n=2178,6453$, and 6282 calcium transient events, respectively; $p<1$ $\times 10^{-20}$ for all conditions, corrected by subtracting shuffled data with randomized peak times; Wilcoxon signedrank test; Fig. 4E). Additionally, we investigated the stability of co-active neuron pairs during wakefulness by comparing the percentage of coincident events within the 1-s window over two consecutive imaging days (Fig. 4F). Percentages ranged from $0 \%$ to $91.2 \%(10.5 \pm 3.0 \%$, mean $\pm \mathrm{SD}$ ) and remained relatively stable across two imaging days (Fig. 4G,H; Pearson's correlation $\rho=0.34$ compared with $\rho=0.06$ for shuffled events; an even higher correlation value of $\rho=0.42$ resulted from calculating co-activity based on estimated SRs; Extended Data Fig. 4-2A). For calcium transients recorded during anesthesia, the correlation of co-activity across days was somewhat lower ( $\rho=0.12$ compared with $\rho=0.05$ for shuffled events; data not shown). To avoid potential confounds by signal contamination from neighboring neurons, we repeated this analysis after excluding nearest neighbors, yielding similar results (Extended Data Fig. 4-3). Together, these findings hint toward functional coupling of neuronal subpopulations in CA3 that is maintained across multiple days.

\section{Discussion}

Our study contributes to the emerging field of in vivo calcium imaging of CA3 pyramidal neurons by establishing longitudinal imaging across days, comparing different behavioral states, and providing calibration in terms of spike patterns underlying the observed calcium transients. We found state-dependent neuronal responses with salient prolonged high-amplitude calcium transients in 
awake mice during locomotion. On the population level, we observed that during wakefulness individual calcium transients are embedded in surrounding network activity, with co-active neuron pairs maintaining their mutual coactivity over days.

Our juxtacellular recordings during anesthesia and the deconvolved calcium transients from awake imaging sessions indicate low mean firing rates but prominent burst events in CA3 pyramidal neurons, in line with previous studies (Henze et al., 2002; Frerking et al., 2005; Wittner and Miles, 2007; Mizuseki et al., 2012; Kowalski et al., 2016; Oliva et al., 2016; Ding et al., 2020). Compared with DG granule cells (Pilz et al., 2016) a much higher fraction of CA3 pyramidal neurons displayed clear calcium transients $(>90 \%$ for all conditions in CA3; for comparison: $<10 \%$ during anesthesia and around $50 \%$ during wakefulness in DG). The mean frequency of calcium transients across the entire population was 6 - to 20 -fold higher in CA3 than in DG, especially during anesthesia. Consistent with the high burstiness of CA3 pyramidal neurons, the vast majority of recorded calcium transients in our ground truth recordings reflected AP bursts rather than individual APs (84\% of event-associated spikes were part of a burst of three or more spikes). The bimodal ISI distribution that we observed during anesthesia (Extended Data Fig. 2-1C) closely resembles previous results during light anesthesia (Kowalski et al., 2016) as well as during sleep and awake behavior (Frerking et al., 2005; Mizuseki et al., 2012). However, it is not straightforward to relate the changes in calcium transient frequency that we observed to changes in AP patterns. Moreover, recent in vivo whole-cell recordings found that theta oscillations were associated with membrane potential hyperpolarization in most CA3 pyramidal neurons (Malezieux et al., 2020), which could imply decreased average firing rates during running. However, theta periods included both resting and running periods and modulatory effects were quite heterogeneous across the CA3 population. Further investigations will be needed to clarify state-dependent modulation of membrane potential dynamics, AP patterns, and cellular calcium signals in CA3.

We estimate that the especially large and prolonged calcium events that we observed were caused by $>30$ APs over $3 \mathrm{~s}$, indicating that a subset of CA3 pyramidal neurons can sustain firing rates of $10 \mathrm{~Hz}$ or higher during running. This spiking level is not too dissimilar from infield firing rates observed in identified CA3 place cells (Mizuseki et al., 2012; Ding et al., 2020). As our experiments were conducted in the dark without salient spatial cues, we can only speculate that these events may relate to place cell or time cell properties. Rather than representing regular spiking, we interpret the large locomotion-related events as presumably reflecting a mixture of regular spikes and bursts at shortened interburst interval compared with resting conditions (note the "bumpy" SRs in the examples in Fig. 3A; see also Epsztein et al., 2011). Previous in vivo studies reported similarly long membrane potential depolarizations in medial entorhinal cortex during anesthesia (Hahn et al., 2012) and in CA1 during awake behavior, the latter termed "hippocampal motifs" that consisted of $\sim 2$-s long AP sequences with above 5$\mathrm{Hz}$ peak rate occurring during foraging behavior (Aghajan et al., 2015). These long-lasting bouts of activity in direct input and output regions of CA3 might be linked to the prolonged high amplitude calcium events that we observed. Further investigations are required in the future to resolve the electrophysiological basis of these special large events during awake running and their relationship to spatial navigation.

Another aspect that warrants further examination is the considerable heterogeneity in functional properties of CA3 pyramidal neurons that has been found along the proximo-distal axis. For example, input resistance and intrinsic excitability are higher in the proximal CA3 region compared with the distal region CA3a, where we performed our recordings (Sun et al., 2017, 2020). Additional factors contributing to the diversity include distinct synaptic inputs from medial entorhinal cortex (Fernandez-Lamo et al., 2019), prefrontal cortex (Rajasethupathy et al., 2015), and the supramammillary nucleus (Lu et al., 2015), as well as differences in dendritic length (Ding et al., 2020) and expression patterns of potassium and hyperpolarization-activated cyclic nucleotide-gated $(\mathrm{HCN})$ channels (Sun et al., 2017; Raus Balind et al., 2019). Heterogeneity also exists along the dorsal-ventral axis (Sun et al., 2017) and between superficial and deep neurons within the pyramidal cell layer (Thompson et al., 2008; Cembrowski and Spruston, 2019). Future calcium imaging studies during awake behavior may help to link this neuronal diversity in CA3 to the propensity of cells to generate large calcium events as well as to specific behaviorally relevant population activity patterns.

Our juxtacellular recordings provide evidence that supra-linear calcium influx might occur with increasing AP numbers, suggesting additional sources that contribute to the mean $\Delta F / F$ values of large events (Fig. $3 D$ ). Additional calcium influx may have been caused by dendritic calcium spikes associated with complex spike bursts (Grienberger et al., 2014; Raus Balind et al., 2019), localized dendritic NMDA spikes (Brandalise et al., 2016), or dendritic plateau potentials induced by supra-linear integration of synaptic inputs (Takahashi and Magee, 2009). Plateau potentials and the associated complex spike bursts have been found to precede place field formation in CA1 neurons and may generally mediate behaviorally relevant plasticity in hippocampal pyramidal neurons (Bittner et al., 2015, 2017; Diamantaki et al., 2018).

The recurrent auto-associative nature of the CA3 network is suitable to support the formation of functional neuronal ensembles (Hopfield, 1982; Nakazawa et al., 2002; Guzman et al., 2016). In our experiments, neurons were more frequently co-active during wakefulness compared with anesthesia (Fig. $4 E$ ), hinting toward the recruitment of CA3 subpopulations during specific behavioral states or in particular sensory environments. Limited by the low temporal resolution of calcium imaging, we could not distinguish whether neurons were co-active on a synaptic time scale (milliseconds) or only on a longer time scale. Yet, we found that these co-active ensembles were relatively stable over consecutive days. Previous calcium 
imaging studies reported unstable space representations in place cells of CA1 and CA3 across days (Rubin et al., 2015; Hainmueller and Bartos, 2020), although some experiments indicate that representations can be stabilized (Kentros et al., 2004; Mankin et al., 2012; Julian et al., 2018). Despite unstable functional representations in single pyramidal neurons, neurons may maintain a stable affiliation to the same engram (Kinsky et al., 2018; Gonzalez et al., 2019) and spatial information could be stably encoded by whole-network activity patterns, based on pairwise co-activity (Stefanini et al., 2020). Across-day stability of a distributed engram, but variable activation patterns of the pyramidal neurons involved, may allow for flexible functional output of hippocampal subpopulations over time (Goode et al., 2020). In our experiments, a subpopulation of CA3 pyramidal neurons displayed large calcium events consistently across days. Furthermore, we found a subset of neurons that were stably co-active with other neurons within the same FOV across days. These results indicate at least some stability in the CA3 neuronal ensemble recruitment processes. Large calcium events associated with complex spike bursting might lead to plasticity in the recurrently connected CA3 network and could support the formation of functional engrams (Raus Balind et al., 2019). The emergence of co-active CA3 ensembles and their relevance for hippocampusdependent behaviors warrant further investigations using longitudinal calcium imaging.

Because of the fundamental importance of the CA3 subfield in the cortico-hippocampal circuitry, we expect a surge of future in vivo CA3 imaging studies that will be facilitated by recent methodological advances. First, although two-photon imaging in DG and CA3 has been achieved with GCaMP indicators (Pilz et al., 2016; Hainmueller and Bartos, 2018), red-shifted calcium indicators may still be beneficial (Pilz et al., 2016; Kondo et al., 2017; Shemetov et al., 2021). Second, pushing excitation wavelengths further into the nearinfrared wavelength is now possible with three-photon microscopy (Ouzounov et al., 2017), with entirely new opportunities for non-invasive hippocampus imaging through the neocortex (Ouzounov et al., 2017; Weisenburger et al., 2019). Finally, the combination of multi-photon imaging with optogenetic manipulation of specific neuronal ensembles, as recently demonstrated in the CA1 region (Robinson et al., 2020), will open new avenues for all-optical interrogation of hippocampal neuronal ensemble dynamics.

\section{References}

Aghajan ZM, Acharya L, Moore JJ, Cushman JD, Vuong C, Mehta MR (2015) Impaired spatial selectivity and intact phase precession in two-dimensional virtual reality. Nat Neurosci 18:121-128.

Bethge P, Carta S, Lorenzo DA, Egolf L, Goniotaki D, Madisen L, Voigt FF, Chen JL, Schneider B, Ohkura M, Nakai J, Zeng H, Aguzzi A, Helmchen F (2017) An R-CaMP1.07 reporter mouse for cell-type-specific expression of a sensitive red fluorescent calcium indicator. PLoS One 12:e0179460.

Bittner KC, Grienberger C, Vaidya SP, Milstein AD, Macklin JJ, Suh J, Tonegawa S, Magee JC (2015) Conjunctive input processing drives feature selectivity in hippocampal CA1 neurons. Nat Neurosci 18:1133-1142.

Bittner KC, Milstein AD, Grienberger C, Romani S, Magee JC (2017) Behavioral time scale synaptic plasticity underlies CA1 place fields. Science 357:1033-1142.

Brandalise F, Carta S, Helmchen F, Lisman J, Gerber U (2016) Dendritic NMDA spikes are necessary for timing-dependent associative LTP in CA3 pyramidal cells. Nat Commun 7:13480.

Buzsáki G (1986) Hippocampal sharp waves: their origin and significance. Brain Res 398:242-252.

Cembrowski MS, Spruston N (2019) Heterogeneity within classical cell types is the rule: lessons from hippocampal pyramidal neurons. Nat Rev Neurosci 20:193-204.

Csicsvari J, Hirase H, Mamiya A, Buzsáki G (2000) Ensemble patterns of hippocampal CA3-CA1 neurons during sharp wave-associated population events. Neuron 28:585-594.

Danielson NB, Turi GF, Ladow M, Chavlis S, Petrantonakis PC, Poirazi P, Losonczy A (2017) In vivo imaging of dentate gyrus mossy cells in behaving mice. Neuron 93:552-559.

Diamantaki M, Coletta S, Nasr K, Zeraati R, Laturnus S, Berens P, Preston-Ferrer P, Burgalossi A (2018) Manipulating hippocampal place cell activity by single-cell stimulation in freely moving mice. Cell Rep 23:32-38.

Ding L, Chen H, Diamantaki M, Coletta S, Preston-Ferrer $P$, Burgalossi A (2020) Structural correlates of CA2 and CA3 pyramidal cell activity in freely-moving mice. J Neurosci 40:5797-5806.

Dombeck DA, Khabbaz AN, Collman F, Adelman TL, Tank DW (2007) Imaging large-scale neural activity with cellular resolution in awake, mobile mice. Neuron 56:43-57.

Dombeck DA, Harvey CD, Tian L, Looger LL, Tank DW (2010) Functional imaging of hippocampal place cells at cellular resolution during virtual navigation. Nat Neurosci 13:1433-1440.

Epsztein J, Brecht M, Lee AK (2011) Intracellular determinants of hippocampal CA1 place and silent cell activity in a novel environment. Neuron 70:109-120.

Fernandez-Lamo I, Gomez-Dominguez D, Sanchez-Aguilera A, Oliva A, Morales AV, Valero M, Cid E, Berenyi A, Menendez de la Prida L (2019) Proximodistal organization of the CA2 hippocampal area. Cell Rep 26:1734-1746.e6.

Fox SE, Ranck JB (1975) Localization and anatomical identification of theta and complex spike cells in dorsal hippocampal formation of rats. Exp Neurol 49:299-313.

Frerking M, Schulte J, Wiebe SP, Stäubli U (2005) Spike timing in CA3 pyramidal cells during behavior: implications for synaptic transmission. J Neurophysiol 94:1528-1540.

Gonzalez WG, Zhang H, Harutyunyan A, Lois C (2019) Persistence of neuronal representations through time and damage in the hippocampus. Science 365:821-825.

Goode TD, Tanaka KZ, Sahay A, Mchugh TJ (2020) An integrated index: engrams, place cells, and hippocampal memory. Neuron 107:805-820.

Grienberger C, Chen X, Konnerth A (2014) NMDA receptor-dependent multidendrite $\mathrm{Ca}\left(2^{+}\right)$spikes required for hippocampal burst firing in vivo. Neuron 81:1274-1281.

Guzman SJ, Schlögl A, Frotscher M, Jonas P (2016) Synaptic mechanisms of pattern completion in the hippocampal CA3 network. Science 353:1117-1123.

Hahn TTG, McFarland JM, Berberich S, Sakmann B, Mehta MR (2012) Spontaneous persistent activity in entorhinal cortex modulates cortico-hippocampal interaction in vivo. Nat Neurosci 15:1531-1538.

Hainmueller T, Bartos M (2018) Parallel emergence of stable and dynamic memory engrams in the hippocampus. Nature 558:292296.

Hainmueller T, Bartos M (2020) Dentate gyrus circuits for encoding, retrieval and discrimination of episodic memories. Nat Rev Neurosci 21:153-168.

Harris KD, Csicsvari J, Hirase H, Dragoi G, Buzsáki G (2003) Organization of cell assemblies in the hippocampus. Nature 424:552556. 
Hartley T, Lever C, Burgess N, O'Keefe J (2013) Space in the brain: how the hippocampal formation supports spatial cognition. Philos Trans R Soc Lond B Biol Sci 369:20120510.

Hemond P, Epstein D, Boley A, Migliore M, Ascoli GA, Jaffe DB (2008) Distinct classes of pyramidal cells exhibit mutually exclusive firing patterns in hippocampal area CA3b. Hippocampus 18:411424.

Henze DA, Wittner L, Buzsáki G (2002) Single granule cells reliably discharge targets in the hippocampal CA3 network in vivo. Nat Neurosci 5:790-795.

Hopfield JJ (1982) Neural networks and physical systems with emergent collective computational abilities. Proc Natl Acad Sci USA 79:2554-2558.

Hunt DL, Linaro D, Si B, Romani S, Spruston N (2018) A novel pyramidal cell type promotes sharp-wave synchronization in the hippocampus. Nat Neurosci 21:985-995.

Jonas P, Major G, Sakmann B (1993) Quantal components of unitary EPSCs at the mossy fibre synapse on CA3 pyramidal cells of rat hippocampus. J Physiol 472:615-663.

Julian JB, Keinath AT, Marchette SA, Epstein RA (2018) The neurocognitive basis of spatial reorientation. Curr Biol Rev 28:10591073.

Kentros CG, Agnihotri NT, Streater S, Hawkins RD, Kandel ER (2004) Increased attention to spatial context increases both place field stability and spatial memory. Neuron 42:283-295.

Kesner RP (2007) Behavioral functions of the CA3 subregion of the hippocampus. Learn Mem 14:771-781.

Kesner RP, Rolls ET (2015) A computational theory of hippocampal function and tests of the theory: new developments. Neurosci Biobehav Rev 48:92-147.

Kinsky NR, Sullivan DW, Mau W, Hasselmo ME, Eichenbaum HB, Kinsky NR, Sullivan DW, Mau W, Hasselmo ME, Eichenbaum HB (2018) Hippocampal place fields maintain a coherent and flexible map across long timescales. Curr Biol 28:3578-3588.

Knierim JJ, Neunuebel JP (2016) Tracking the flow of hippocampal computation: pattern separation, pattern completion, and attractor dynamics. Neurobiol Learn Mem 129:38-49.

Kondo M, Kobayashi K, Ohkura M, Nakai J, Matsuzaki M (2017) Two-photon calcium imaging of the medial prefrontal cortex and hippocampus without cortical invasion. Elife 6:e26839.

Kowalski J, Gan J, Jonas P, Pernía-Andrade AJ (2016) Intrinsic membrane properties determine hippocampal differential firing pattern in vivo in anesthetized rats. Hippocampus 26:668-682.

Langer D, van 't Hoff M, Keller AJ, Nagaraja C, Pfäffli OA, Göldi M, Kasper H, Helmchen F (2013) HelioScan: a software framework for controlling in vivo microscopy setups with high hardware flexibility, functional diversity and extendibility. J Neurosci Methods 215:3852.

Lee D, Lin BJ, Lee AK (2012) Hippocampal place fields emerge upon single-cell manipulation of excitability during behavior. Science 337:849-853.

Leutgeb S, Leutgeb JK, Treves A, Moser MB, Moser El (2004) Distinct ensemble codes in hippocampal areas CA3 and CA1. Science 305:1295-1298.

Lu L, Igarashi KM, Witter MP, Moser El, Moser MB (2015) Topography of place maps along the CA3-to-CA2 axis of the hippocampus. Neuron 87:1078-1092.

Lütcke H, Margolis DJ, Helmchen F (2013) Steady or changing? Long-term monitoring of neuronal population activity. Trends Neurosci 36:375-384.

Malezieux M, Kees AL, Mulle C (2020) Theta oscillations coincide with sustained hyperpolarization in CA3 pyramidal cells, underlying decreased firing. Cell Rep 32:107868.

Mankin EA, Sparks FT, Slayyeh B, Sutherland RJ, Leutgeb S, Leutgeb JK (2012) Neuronal code for extended time in the hippocampus. Proc Natl Acad Sci USA 109:19462-19467.

Marissal T, Bonifazi P, Picardo MA, Nardou R, Petit LF, Baude A, Fishell GJ, Ben-Ari Y, Cossart R (2012) Pioneer glutamatergic cells develop into a morpho-functionally distinct population in the juvenile CA3 hippocampus. Nat Commun 3:1316.
Menendez De La Prida L, Huberfeld G, Cohen I, Miles R (2006) Threshold behavior in the initiation of hippocampal population bursts. Neuron 49:131-142.

Miles R, Wong RKS (1983) Single neurones can initiate synchronized population discharge in the hippocampus. Nature 306:371-373.

Mizuseki K, Royer S, Diba K, Buzsáki G (2012) Activity dynamics and behavioral correlates of $\mathrm{CA} 3$ and $\mathrm{CA} 1$ hippocampal pyramidal neurons. Hippocampus 22:1659-1680.

Nakazawa K, Quirk MC, Chitwood RA, Watanabe M, Yeckel MF, Sun LD, Kato A, Carr CA, Johnston D, Wilson MA, Tonegawa S (2002) Requirement for hippocampal CA3 NMDA receptors in associative memory recall. Science 297:211-218.

Ohkura M, Sasaki T, Kobayashi C, Ikegaya Y, Nakai J (2012) An improved genetically encoded red fluorescent $\mathrm{Ca} 2+$ indicator for detecting optically evoked action potentials. PLoS One 7:e39933.

Oliva A, Fernández-Ruiz A, Buzsáki G, Berényi A (2016) Spatial coding and physiological properties of hippocampal neurons in the Cornu Ammonis subregions. Hippocampus 26:1593-1607.

Ouzounov DG, Wang T, Wang M, Feng DD, Horton NG, CruzHernández JC, Cheng YT, Reimer J, Tolias AS, Nishimura N, Xu C (2017) In vivo three-photon imaging of activity of GCaMP6-labeled neurons deep in intact mouse brain. Nat Methods 14:388-390.

Pilz GA, Carta S, Stäuble A, Ayaz A, Jessberger S, Helmchen F (2016) Functional imaging of dentate granule cells in the adult mouse hippocampus. J Neurosci 36:7407-7414.

Pilz GA, Bottes S, Betizeau M, Jörg DJ, Carta S, Simons BD, Helmchen F, Jessberger $S$ (2018) Live imaging of neurogenesis in the adult mouse hippocampus. Science 359:658-662.

Rajasethupathy P, Sankaran S, Marshel JH, Kim CK, Ferenczi E, Lee SY, Berndt A, Ramakrishnan C, Jaffe A, Lo M, Liston C, Deisseroth $\mathrm{K}$ (2015) Projections from neocortex mediate top-down control of memory retrieval. Nature 526:653-659.

Rashid SK, Pedrosa V, Dufour MA, Moore JJ, Delatorre RG, Poirazi P, Clopath C, Basu J (2020) The dendritic spatial code: branchspecific place tuning and its experience-dependent decoupling. bioRxiv 2020.01.24.916643.

Raus Balind S, Magó Á, Ahmadi M, Kis N, Varga-Németh Z, Lörincz A, Makara JK (2019) Diverse synaptic and dendritic mechanisms of complex spike burst generation in hippocampal CA3 pyramidal cells. Nat Commun 10:1859.

Rebola N, Carta M, Mulle C (2017) Operation and plasticity of hippocampal CA3 circuits: implications for memory encoding. Nat Rev Neurosci 18:208-220.

Robinson NTM, Descamps LAL, Russell LE, Buchholz MO, Bicknell BA, Antonov GK, Lau JYN, Nutbrown R, Schmidt-Hieber C, Häusser M (2020) Targeted activation of hippocampal place cells drives memory-guided spatial behavior. Cell 183:1586-1599.e10.

Rolls ET (2007) An attractor network in the hippocampus: theory and neurophysiology. Learn Mem 14:714-731.

Rolls ET (2016) Pattern separation, completion, and categorisation in the hippocampus and neocortex. Neurobiol Learn Mem 129:4-28.

Rubin A, Geva N, Sheintuch L, Ziv Y (2015) Hippocampal ensemble dynamics timestamp events in long-term memory. Elife 4:e12247.

Rupprecht P, Carta S, Hoffmann A, Echizen M, Blot A, Kwan AC, Dan Y, Hofer SB, Kitamura K, Helmchen F, Friedrich RW (2021) A database and deep learning toolbox for noise-optimized, generalized spike inference from calcium imaging. Nat Neurosci. Advance online publication. Retrieved Aug 2, 2021. doi:10.1038/s41593-021-00895-5.

Schindelin J, Arganda-Carreras I, Frise E, Kaynig V, Longair M, Pietzsch T, Preibisch S, Rueden C, Saalfeld S, Schmid B, Tinevez JY, White DJ, Hartenstein V, Eliceiri K, Tomancak P, Cardona A (2012) Fiji: an open-source platform for biological-image analysis. Nat Methods 9:676-682.

Shemetov AA, Monakhov MV, Zhang Q, Canton-Josh JE, Kumar M, Chen M, Matlashov ME, Li X, Yang W, Nie L, Shcherbakova DM, Kozorovitskiy Y, Yao J, Ji N, Verkhusha VV (2021) A near-infrared genetically encoded calcium indicator for in vivo imaging. Nat Biotechnol 39:368-377. 
Stefanini F, Kushnir L, Jimenez JC, Jennings JH, Woods NI, Stuber GD, Kheirbek MA, Hen R, Fusi S (2020) A distributed neural code in the dentate gyrus and in CA1. Neuron 107:703-716.

Sun Q, Sotayo A, Cazzulino AS, Snyder AM, Denny CA, Siegelbaum SA (2017) Proximodistal heterogeneity of hippocampal CA3 pyramidal neuron intrinsic properties, connectivity, and reactivation during memory recall. Neuron 95:656-672.e3.

Sun Q, Jiang YQ, Lu MC (2020) Topographic heterogeneity of intrinsic excitability in mouse hippocampal CA3 pyramidal neurons. J Neurophysiol 124:1270-1284.

Takahashi H, Magee JC (2009) Pathway interactions and synaptic plasticity in the dendritic tuft regions of CA1 pyramidal neurons. Neuron 62:102-111.

Thompson CL, Pathak SD, Jeromin A, Ng LL, MacPherson CR, Mortrud MT, Cusick A, Riley ZL, Sunkin SM, Bernard A, Puchalski RB, Gage FH, Jones AR, Bajic VB, Hawrylycz MJ, Lein ES (2008) Genomic anatomy of the hippocampus. Neuron 60:1010-1021.
Weisenburger S, Tejera F, Demas J, Chen B, Manley J, Sparks FT, Martínez Traub F, Daigle T, Zeng H, Losonczy A, Vaziri A (2019) Volumetric $\mathrm{Ca}_{2}{ }^{+}$imaging in the mouse brain using hybrid multiplexed sculpted light microscopy. Cell 177:1050-1066.

Wittner L, Miles R (2007) Factors defining a pacemaker region for synchrony in the hippocampus. J Physiol 584:867-883.

Yang W, Chini M, Pöpplau JA, Formozov A, Dieter A, Piechocinski P, Rais C, Morellini F, Sporns O, Hanganu-Opatz IL, Wiegert JS (2021) Anesthetics fragment hippocampal network activity, alter spine dynamics and affect memory consolidation. PLoS Biol 19: e3001146.

Ziv Y, Burns LD, Cocker ED, Hamel EO, Ghosh KK, Kitch LJ, El Gamal A, Schnitzer MJ (2013) Long-term dynamics of CA1 hippocampal place codes. Nat Neurosci 16:264-266.

Zucca S, Griguoli M, Malézieux M, Grosjean N, Carta M, Mulle C (2017) Control of spike transfer at hippocampal mossy fiber synapses in vivo by $\mathrm{GABA}_{A}$ and $\mathrm{GABA}_{B}$ receptor-mediated inhibition. J Neurosci 37:587-598. 